\title{
Neuorientierung bei globaler Unsicherheit
}

Die verarbeitende Industrie steht vor großen Herausforderungen, denn die Verwerfungen im Wirtschaftsgeschehen parallel zu und in Folge der Corona-Krise haben Quellen für unternehmerische Risiken noch deutlicher werden lassen. Gestörte Lieferketten, nicht durchgängige Digitalisierung, Einsatz neuer Technologien und Geschäftsmodelle, Erwachsen neuer Konkurrenz aus Schwellenländern und nicht zuletzt politische Risiken für den weltumspannenden Handel führen zu einer global gefühlten Unsicherheit.

Für produzierende Unternehmen bedeutet das, dass einer permanenten Analyse des eigenen Risikoprofils auch regelmäßig eine technologisch, methodisch und strategisch geprägte Neuorientierung folgen muss. Das gilt umso mehr, als dass sich die Erwartung von Partnern und Kunden in Bezug auf Informationsverfügbarkeit und nahtlose Zusammenarbeit signifikant erhöht hat.

Die Unternehmen, die zukünftig erfolgreich sein werden, sind diejenigen, die von der Hyperkonnektivität ihrer Kunden profitieren. Die Cloud ermöglicht es der Fertigungsindustrie, ihre globalen Programme zu koordinieren, die Ausführung zu beschleunigen und intern besser zusammenzuarbeiten. Die innovativsten Unternehmen gehen sogar noch weiter, indem sie das gesamte Ökosystem von Partnern mit direktem Zugang zu ihrer Herstellung, Produktion oder Lieferkette einbetten. Digitale Plattformen, die den Kunden in den Mittelpunkt eines Unternehmens stellen sowie Vertriebs-, Service- und Marketingaufgaben integrieren, helfen den Herstellern, Risiken früher zu erkennen und Herausforderungen schneller zu meistern. Für Unternehmen, die diesen Prozess noch nicht begonnen haben, ist es höchste Zeit, sich diesen Herausforderungen zu stellen - wenn es auch eine Neuorientierung bei globaler Unsicherheit bedeutet.

Ihre ZWF-Herausgeber und-Redaktion

KorrekturPro - Experten für Korrektur, Formatierung und Satz.

Ihr zuverlässiger Partner für Textkorrektur und -lektorat, Grafik, Diagramme und Formeln. Wir begleiten Sie von der Rohfassung bis zur Druckvorstufe.

Korrektur und Lektorat

Rechtschreibung, Zeichensetzung, Ausdruck, Stil und Grammatik: von der Rohfassung bis zum druckreifen Manuskript

Formatierung und Layout

Satz, Layout, Fuß- und Endnoten, Formeln, Index und andere Verzeichnisse, Druckformatvorlagen gemäß verlagsspezifischer Anforderungen
Coaching und Recherche

Recherche, Arbeitsplanung, korrektes Zitieren, wissenschaftliches Bibliographieren

Unterstützung für Nicht-

Muttersprachler

Wissenschaftlicher Sprachstil, Vereinheitlichungen, Fachsprachen und -vokabular

Alle Texte, alle Formate

Wissenschaftliche Arbeiten, Monographien, Editionen, Konferenzbände, Jahresberichte, Präsentationen und Poster
KorrekturPro • Berlin

Korrektur \& Formatierung

Text, Grafik, Diagramme, Formeln, Druckvorstufe

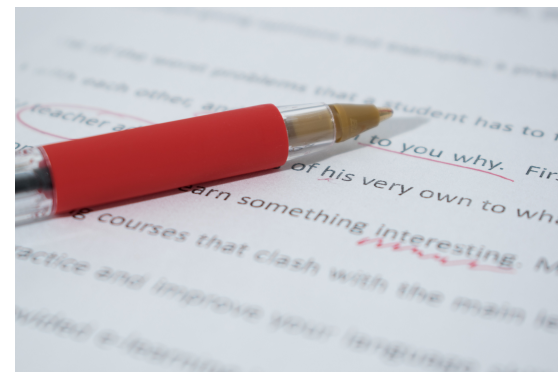

KorrekturPro 